\title{
Simulation study of the phase behavior of a planar Maier-Saupe nematogenic liquid
}

\author{
E. Lomba, C. Martín, and N. G. Almarza \\ Instituto de Química Física Rocasolano, CSIC, Serrano 119, E-28006 Madrid, Spain \\ F. Lado \\ Department of Physics, North Carolina State University, Raleigh, North Carolina 27695-8202, USA
}

(Received 17 January 2005; published 22 April 2005)

\begin{abstract}
Using extensive Monte Carlo simulations and a simple approximation in density functional theory, we study the phase behavior of a fluid of nematogenic molecules with centers of mass constrained to lie in a plane but with axes free to rotate in any direction, both with and without an external disorienting field perpendicular to the plane. We find that simulation predicts the existence of an order-disorder phase transition belonging to the Berezinskii-Kosterlitz-Thouless type, along with a low temperature gas-liquid transition. In contrast to the simulation results, density functional theory predicts a first-order orientational phase transition coupled continuously with a first-order gas-liquid transition. The approximate theoretical approach qualitatively reproduces the field dependence of the order-disorder and gas-liquid transitions but is far from quantitative.
\end{abstract}

DOI: 10.1103/PhysRevE.71.046132

PACS number(s): 64.60.Cn, 61.20.Gy

\section{INTRODUCTION}

In previous papers [1], the authors presented a study of the structure and thermodynamics of a fluid composed of particles interacting via a Maier-Saupe potential [2] and constrained to lie in a plane. Such a system, which is a continuous version of the lattice $\mathrm{RP}^{2}$ model studied by Kunz and Zumbach [3], was earlier studied by Sokolovska, Sokolovskii, and Holovko with the presence of both finite [4] and infinite [5] disorienting fields $\mathbf{W}_{0}$ perpendicular to the plane. The external field tends to align the particles' molecular axes perpendicular to itself, whereby an in-plane orderdisorder transition is favored, as confirmed by the results reported in [1]. It was noted in those works that this system very likely undergoes a defect-mediated order-disorder transition of the Berezinskii-Kosterliz-Thouless (BKT) type $[6,7]$, in which the ordered phases lack true long-range order and the corresponding order parameters vanish in the thermodynamic limit. Additionally, the Maier-Saupe interaction induces a net attraction between the particles that can lead to a condensation transition. It is the principal aim of this paper to investigate both the order-disorder and the gas-liquid transitions in this planar nematogenic fluid, especially the influence of the external disorienting field up to the limiting case of planar rotors, $W_{0} \rightarrow \infty$. To that end, we make use of extensive Monte Carlo (MC) simulations and a simple density functional theory. In particular, for the study of the gas-liquid transition we introduce an efficient simulation technique based on the Wang-Landau method [8] for the calculation of the density of states in lattice models. The method proposed here is especially suited to deal with hard-core systems and allows for a straightforward implementation of the finite size scaling (FSS) techniques required for a proper accounting of the long-range fluctuations associated with the presence of critical points.

Let us recall the essential features of the interaction model under review. The total potential energy $U$ of the $N$-molecule system in a particular configuration is given by

$$
U=\sum_{j} u_{z}\left(\omega_{j}\right)+\sum_{i<j} u_{\mathrm{HS}}\left(r_{i j}\right)+\sum_{i<j} u\left(r_{i j}, \omega_{i}, \omega_{j}\right)
$$

where $\omega=(\theta, \phi)$ specifies the orientation of a molecular nematic axis, which is free to rotate in three dimensions, referred to the $z$ axis perpendicular to the system plane. The energies here are the external disorienting potential in the $z$ direction,

$$
u_{z}(\omega)=W_{0} P_{2}(\cos \theta),
$$

which for $W_{0}>0$ favors molecular axes lying in the system plane, the hard-sphere potential $u_{\mathrm{HS}}(r)$ for spheres of diameter $\sigma$, and an attractive potential between a pair of molecules that tends to align their mutual nematic axes,

$$
u\left(r, \omega_{1}, \omega_{2}\right)=-K u_{0}(r) P_{2}\left(\cos \theta_{12}\right) \quad \text { with } K>0,
$$

with

$$
u_{0}(r)= \begin{cases}\frac{e^{-\kappa(r-\sigma)}}{r / \sigma}-\frac{e^{-\kappa(R-\sigma)}}{R / \sigma} & \text { for } \sigma<r<R, \\ 0 & \text { for } r>R .\end{cases}
$$

Here, $P_{2}(x)=\left(3 x^{2}-1\right) / 2$ is the Legendre polynomial of order $2, \theta_{12}$ is the angle between the axes of molecules 1 and 2, and $\kappa$ is a range parameter. The potential $u_{0}(r)$ is truncated and shifted at $R=4 \sigma$ for the convenience of the simulation; this will obviously affect the system properties as compared to those of the untruncated potential studied in [1]. However, the phase behavior will be qualitatively similar, with the transition temperatures now being somewhat lower.

In the calculation, we define the reduced temperature as $T^{*}=k_{B} T / K=1 / \beta K$ and the reduced external field as $W_{0}^{*}$ $=W_{0} / K$, where $T$ is the absolute temperature, $k_{B}$ is Boltzmann's constant, and $K$ is the coupling strength in Eq. (3); we set the range parameter to $\kappa=\sigma^{-1}$.

The rest of the paper is organized as follows. In the next section we describe the details of the density functional theory. Section III is devoted to a presentation of the details of the simulation procedure, in particular those aspects that 
concern the search for the gas-liquid transition. (The cluster algorithms utilized for an efficient sampling in the neighborhood of the order-disorder transition can be found explained in full detail in the literature [9-13].) In Sec. IV we present and discuss our most significant results.

\section{DENSITY FUNCTIONAL THEORY}

For a hard-core system such as the one we are dealing with, it is convenient to construct the free energy functional as a perturbation around the hard-disk reference system [14]. Following this prescription, one gets for the free energy functional $F$,

$$
F[\rho]=F_{0}[\rho]+F_{1}[\rho]+F_{\text {field }}[\rho],
$$

where the hard-disk reference part $F_{0}$ can in turn be expressed in terms of an ideal and an excess component,

$$
F_{0}[\rho]=F_{0}^{\mathrm{id}}[\rho]+F_{0}^{\mathrm{ex}}[\rho] .
$$

Here the excess part can be approximated using Luding's equation of state for hard disks [15], which once integrated leads to the following excess free energy per particle:

$$
\frac{\beta F_{0}^{\mathrm{ex}}}{N}=\frac{1}{64}\left(\frac{421-837 \eta+414 \eta^{2}}{6(1-\eta)^{3}}-56 \ln (1-\eta)\right)-\frac{421}{384},
$$

where $\eta=\pi \rho \sigma^{2} / 4$ is the hard-disk packing fraction. As to the ideal part, its contribution per particle reads

$$
\frac{\beta F_{0}^{\mathrm{id}}}{N}=\ln \rho \Lambda^{2}-1+\int d \omega \alpha(\omega) \ln [4 \pi \alpha(\omega)],
$$

where $\alpha(\omega)$ is the one-particle angular distribution function normalized to unity and $\Lambda$ is the de Broglie thermal wavelength. The external field contribution per particle reads

$$
\frac{\beta F_{\text {field }}}{N}=\frac{1}{2} \beta W_{0}\left(3\left\langle x^{2}\right\rangle-1\right),
$$

with $x=\cos \theta$. To proceed further one must minimize the free energy functional with respect to the one-particle orientational distribution $\alpha(\omega)$; the perturbative part of the free energy functional, $F_{1}$, must be approximated in some fashion. Two widely used approaches are discussed below.

\section{A. Mean field approximation}

The simplest choice one can make to estimate the contribution to the free energy of the angle-dependent part of the interaction is the mean field (MF) approach, according to which

$$
\frac{\beta F_{1}}{N}=\frac{1}{2} \rho \int d \mathbf{r}_{12} d \omega_{1} d \omega_{2} \alpha\left(\omega_{1}\right) \alpha\left(\omega_{2}\right) \beta u\left(r_{12}, \omega_{1}, \omega_{2}\right) .
$$

This equation is adequate for phases with long-range order, but in the isotropic phase-in which $\alpha(\omega)=1 / 4 \pi$-it yields $F_{1}=0$ due to the symmetry of the interaction Eq. (3). In any case, this can be a good starting point and in ferromagnetic systems has proven to be a relatively accurate approximation as far as the phase diagram is concerned [16]. In order to perform the minimization with respect to $\alpha(\omega)$ one could expand this function in terms of orthogonal functions and then minimize the functional with respect to the expansion coefficients. This is, however, a numerically ill-conditioned problem which can be bypassed by using a predefined functional form for $\alpha(\omega)$. Here we can resort to a parametrized $\alpha(\omega)$ which retains the functional form that one encounters for noninteracting particles in the presence of the disorienting field $\mathbf{W}_{0}$ and a transverse field $\mathbf{A}_{0}$ of the type used in [1]. One has then

$$
\alpha(\theta, \phi)=\frac{\exp \left(-w \cos ^{2} \theta+a \cos ^{2} \phi\right)}{C_{\theta} C_{\phi}},
$$

where $w$ and $a$ will be the variational parameters of the free energy functional. The normalization constants are given by

$$
C_{\theta}=\int_{-1}^{1} e^{-w x^{2}} d x=\sqrt{\frac{\pi}{w}} \operatorname{erf}(\sqrt{w})
$$

and

$$
C_{\phi}=\int_{0}^{2 \pi} e^{a \cos ^{2} \phi} d \phi=2 \pi e^{a / 2} I_{0}(a / 2),
$$

where $\operatorname{erf}(x)$ is the error function and $I_{0}(x)$ the zeroth-order modified Bessel function of the first kind. Equation (9) can now be integrated to give

$$
\frac{\beta F_{0}^{\text {id }}}{N}=\ln \rho \Lambda^{2}-1+\ln \left(\frac{4 \pi}{C_{\theta} C_{\phi}}\right)-w\left\langle x^{2}\right\rangle+a\left\langle y^{2}\right\rangle,
$$

where $x=\cos \theta, y=\cos \phi$, and

$$
\begin{gathered}
\left\langle x^{2}\right\rangle=\frac{1}{C_{\theta}}\left(-\frac{e^{-w}}{w}+\frac{\sqrt{\pi} \operatorname{erf}(\sqrt{w})}{2 w^{2 / 3}}\right), \\
\left\langle y^{2}\right\rangle=\frac{I_{0}(a / 2)+I_{1}(a / 2)}{2 I_{0}(a / 2)},
\end{gathered}
$$

with $I_{1}(x)$ being the first-order modified Bessel function of the first kind. In principle, one can use higher powers of the angular variables in Eq. (12), but in the case of a MF approach one finds that the quadratic terms are the only ones needed.

The perturbation part can also be evaluated explicitly, yielding

$$
\begin{aligned}
\frac{\beta F_{1}}{N}= & -\frac{\pi \beta K \rho \sigma^{2}}{2 \kappa \sigma}\left(1-\frac{R(2+\kappa R)-\kappa \sigma^{2}}{2 R} \exp [-\kappa(R-\sigma)]\right) \\
& \times\left\{3\left\langle x^{2}\right\rangle^{2}+3\left(1-\left\langle x^{2}\right\rangle\right)^{2}\left[\left\langle y^{2}\right\rangle^{2}+\left(1-\left\langle y^{2}\right\rangle\right)^{2}\right]-1\right\} .(18
\end{aligned}
$$

Now the functional (6) with Eqs. (8), (15), (10), and (18) can be minimized with respect to the parameters $w$ and $a$ using a simple conjugate gradient method [17]. Once this is performed, one can also obtain the excess contribution to the internal energy $U^{\text {ex }}$, which in this approximation is simply 


$$
\beta U^{\mathrm{ex}} / N=\beta F_{1} / N
$$

and the pressure $P$, which is given by

$$
\beta p / \rho=z_{\mathrm{HD}}+\beta F_{1} / N,
$$

where the hard-disk compressibility factor is [15]

$$
z_{\mathrm{HD}}=1+2 \eta\left(\frac{1-7 \eta / 16}{(1-\eta)^{2}}-\frac{\eta^{3}}{128(1-\eta)^{4}}\right)
$$

\section{B. Modified mean field approximation}

As noted earlier, when the system is isotropic the MF excess contribution to the free energy vanishes due to the symmetry of the potential. In order to avoid this shortcoming, Teixeira and Telo da Gama [18] proposed the use of a low-density approximation in the construction of the excess free energy functional, leading to

$$
\begin{aligned}
\frac{\beta F_{1}}{N}= & \frac{1}{2} \rho \int d \mathbf{r}_{12} d \omega_{1} d \omega_{2} \alpha\left(\omega_{1}\right) \alpha\left(\omega_{2}\right) \\
& \times\left\{1-\exp \left[-\beta u\left(r_{12}, \omega_{1}, \omega_{2}\right)\right]\right\} .
\end{aligned}
$$

Now, however, the integral in Eq. (22) cannot be evaluated analytically even in the simpler case of a Gaussian approximation for the one-particle angular distribution function (12). Moreover, since Eq. (22) is highly nonlinear in $x^{2}$ and $y^{2}$, one should in this case go beyond the quadratic approximation for $\alpha(\omega)$. We have thus used

$$
\begin{aligned}
\alpha(\theta, \phi)= & \frac{1}{C_{\theta} C_{\phi}} \exp \left(-w \cos ^{2} \theta-w^{\prime} \cos ^{4} \theta+a \cos ^{2} \phi\right. \\
& \left.+a^{\prime} \cos ^{4} \phi\right),
\end{aligned}
$$

with $C_{\theta}$ and $C_{\phi}$ defined by normalization conditions analogous to (13) and (14), which must be evaluated numerically. Equation (15) now becomes

$$
\begin{aligned}
\frac{\beta F_{0}^{\mathrm{id}}}{N}= & \ln \rho \Lambda^{3}-1+\ln \left(\frac{4 \pi}{C_{\theta} C_{\phi}}\right)-w\left\langle x^{2}\right\rangle-w^{\prime}\left\langle x^{4}\right\rangle+a\left\langle y^{2}\right\rangle \\
& +a^{\prime}\left\langle x^{4}\right\rangle
\end{aligned}
$$

and the moments must also be calculated by numerical integration. The multidimensional integrals involved in this process can be evaluated using Gaussian quadrature rules based on the special orthogonal polynomials developed in [1] or by means of standard Gauss-Legendre and Gauss-Chebyshev quadratures. Finally, the excess internal energy derived from Eq. (22) is given by

$$
\begin{aligned}
\frac{\beta U^{\mathrm{ex}}}{N}= & \frac{1}{2} \rho \int d \mathbf{r}_{12} d \omega_{1} d \omega_{2} \alpha\left(\omega_{1}\right) \alpha\left(\omega_{2}\right) \beta u\left(r_{12}, \omega_{1}, \omega_{2}\right) \\
& \times \exp \left[-\beta u\left(r_{12}, \omega_{1}, \omega_{2}\right)\right]
\end{aligned}
$$

while for the pressure Eq. (20) remains valid.

\section{SIMULATION METHODOLOGY}

Various aspects of the simulation technique merit discussion in some detail. On the one hand, we will be dealing here with a rather peculiar system in which the "ordered phases" seem to lack a true long-range order but the correlation length and the susceptibility at a given density $\rho$ diverge for any temperature below the transition temperature $T_{\mathrm{KT}}[\rho]$. On the other hand, for sufficiently low temperatures the orientational average of the Maier-Saupe interaction leads to a sufficiently long-ranged net attraction that induces a gas-liquid transition coupled with the order-disorder transition. In what follows we will first briefly review the simulation techniques needed for an analysis of the order-disorder transition and then present our method of choice to explore the lowtemperature condensation.

\section{A. The orientational transition}

As noted in [1], the order-disorder transition will take place at moderate to high densities for sufficiently low temperatures in the absence of external field or at slightly higher temperatures when the disorienting field $\mathbf{W}_{0}$ is turned on. For these systems we apply a combination of cluster and singleparticle moves. The cluster algorithm used was developed following the ideas of Swendsen and Wang [11] and can be found in full detail in Ref. [13] applied to an off-lattice Heisenberg system. We note that in the present application some of the reflection moves will be redundant, given the symmetry of the interaction, but this hardly affects the results. If needed, the algorithm could easily be adapted to the symmetry of the Maier-Saupe interaction following the prescription of Priezjev and Pelcovits [12]. Additionally, when $\mathbf{W}_{0} \neq \mathbf{0}$ the reflection planes must contain $\mathbf{W}_{0}$, whereby the cluster moves are purely bidimensional. This implies that the cluster sampling will not be completely ergodic in this case, but this does not pose any difficulty since cluster moves are complemented with single-particle moves.

As to the single-particle orientational moves for $\mathbf{A}_{0}=\mathbf{0}$ cases, we have implemented an efficient algorithm which guarantees that each and every particle move is accepted. The main lines of the algorithm are as follows.

(1) Randomly choose a particle $j$ whose orientation is described by a unit vector $\hat{\mathbf{s}}_{j}$ of components $s_{j}^{\alpha}(\alpha=x, y, z)$.

(2) Determine the orientation $\hat{\mathbf{s}}_{j}^{\text {min }}$ for which the interaction of the chosen particle with the remaining particles of the system corresponds to a minimum. This step is easily carried out for our system if one notices that the relevant energy

$$
\beta u_{j}=-\frac{3}{2} \beta K \sum_{i} u_{0}\left(r_{i j}\right)\left(\hat{\mathbf{s}}_{i} \cdot \hat{\mathbf{s}}_{j}\right)^{2}+\frac{3}{2} \beta W_{0}\left(\hat{\mathbf{s}}_{j} \cdot \hat{\mathbf{W}}_{0}\right)^{2}
$$

(where the additive $1 / 2$ terms from the $P_{2}$ polynomials have been dropped since they do not affect the orientational move) can be recast in matrix form as

$$
\beta u_{j}=\hat{\mathbf{s}}_{j} \cdot\left(\mathbf{K}^{j}+\mathbf{W}\right) \cdot \hat{\mathbf{s}}_{j}
$$

where

$$
K_{\alpha \beta}^{j}=-\frac{3}{2} \beta K \sum_{i} u_{0}\left(r_{i j}\right) s_{i}^{\alpha} s_{i}^{\beta}
$$




$$
W_{\alpha \beta}=\frac{3}{2} \beta W_{0} \delta_{\alpha z} \delta_{\beta z}
$$

with $\alpha, \beta=x, y, z$. In the above equations $\hat{\mathbf{W}}_{0}$ is a unit vector in the direction of the field, in this case the $z$ axis. By diagonalization we have

$$
\beta u_{j}=\sum_{\alpha} \lambda_{\alpha}\left(s_{j}^{\prime \alpha}\right)^{2}
$$

where the $\lambda_{\alpha}$ are the eigenvalues of $\mathbf{K}^{j}+\mathbf{W}$ and $\hat{\mathbf{s}}_{j}^{\prime}=\mathbf{U} \cdot \hat{\mathbf{s}}_{j}$, with $\mathbf{U}$ being the matrix formed by the eigenvectors of $\mathbf{K}^{j}$ $+\mathbf{W}$. Thus, minimizing (30) with the constraint that $\hat{\mathbf{s}}_{j}$ is normalized, one finds that the minimum energy corresponds to the minimum eigenvalue $\lambda_{\min }$ of $\mathbf{K}^{j}+\mathbf{W}$ and the corresponding orientation is given by $\mathbf{U}^{-1} \cdot \hat{\mathbf{s}}_{j}^{\prime m i n}$.

(3) With the energy minimum calculated, pick a random orientation described by a vector $\hat{\mathbf{s}}_{j}$ and evaluate the ratio

$$
\xi=\frac{\exp \left(-\beta u_{j}\right)}{\exp \left(-\beta u_{j}^{\min }\right)}=\exp \left[-\left(\beta u_{j}-\lambda_{\min }\right)\right] .
$$

(4) Finally, generate a uniformly distributed random number $\chi, 0 \leqslant \chi \leqslant 1$, and accept the new orientation if $\chi \leqslant \xi$; otherwise, return to the previous step.

The procedure outlined above combined with the cluster moves considerably improves the orientational sampling.

The orientational order of the sample is monitored through the eigenvalues of Saupe's tensor in three dimensions

$$
Q_{\alpha \beta}^{3 \mathrm{D}}=\frac{1}{2 N} \sum_{i=1}^{N}\left\langle 3 s_{i}^{\alpha} s_{i}^{\beta}-\delta_{\alpha \beta}\right\rangle \quad \text { with } \alpha, \beta=x, y, z .
$$

The eigenvalues will be denoted here by $\lambda_{-}, \lambda_{0}, \lambda_{+}$. As the eigenvalues of a traceless tensor, they satisfy $\lambda_{-}+\lambda_{0}+\lambda_{+}=0$. The largest of the three eigenvalues, $\lambda_{+}$, defines the orientational order parameter $S=\lambda_{+}$. The size dependence of this quantity, and in particular that of Binder's cumulants constructed with it, will be crucial in determining the location of the transition temperature. To that purpose, we have simulated various sample sizes, ranging from 100 to 6400 particles. Note that for infinite disorienting fields the molecular axes will lie on the plane and so the two-dimensional version of Saupe's tensor must be employed, namely,

$$
Q_{\alpha \beta}^{2 \mathrm{D}}=\frac{1}{N} \sum_{i=1}^{N}\left\langle 2 s_{i}^{\alpha} s_{i}^{\beta}-\delta_{\alpha \beta}\right\rangle \quad \text { with } \alpha, \beta=x, y .
$$

Now, in order to perform the FSS analysis, one should have access to the calculation of certain quantities (order parameters, susceptibilities, etc.) for temperatures other than those for which the simulations have been run. Taking into account that the cost of simulating large samples is not negligible, we have resorted to the optimized histogram reweighting technique to produce estimates of temperature-independent quantities from the recombination of energy histograms of the desired quantities, in particular the order parameters. For this purpose we have followed the prescription of Ferrenberg and Swendsen [9] and Holm and Janke [10]. A detailed description of the procedure can be found in Ref. [13].

\section{B. Gas-liquid equilibrium}

Recently, Wang and Landau [8] proposed an efficient method to evaluate the density of states of lattice models. Following their ideas, a number of papers have appeared in which the method has been generalized to cope with offlattice simulations [19-21]. In this contribution, we propose a technique for the study of phase equilibria that is built on the ideas underlying Wang and Landau's method. In what follows we will sketch the basics of the simulation procedure for a simple fluid, the generalization to other systems being straightforward.

The classical partition function of a simple fluid in the canonical ensemble is given by

$$
Q_{N V T}=\frac{V^{N}}{\Lambda^{d N} N !} \int d \mathbf{s}^{N} e^{-\beta U}
$$

where $V$ is the "volume," $N$ is the number of particles, $d$ is the dimensionality of the space, $\mathbf{s}^{N}$ represents the coordinates (reduced with the system size) of the $N$ particles of the system, and $U$ is the potential energy, which depends on $\mathbf{s}^{N}$ and $V$. The partition function is related to the Helmholtz free energy $F$ by

$$
F=-k_{B} T \ln Q_{N V T} .
$$

The free energy $F$ can be written as a sum of ideal $F^{\text {id }}$ and excess $F^{\text {ex }}$ contributions, with

$$
\begin{gathered}
F^{\mathrm{id}}=-k_{B} T \ln \left(\frac{V^{N}}{N ! \Lambda^{d N}}\right), \\
F^{\mathrm{ex}}=-k_{B} T \ln \left(\int d \mathbf{s}^{N} e^{-\beta U}\right) .
\end{gathered}
$$

When performing computer simulation of certain problems it is useful to sample over different particle numbers in conditions of fixed temperature and volume; this is the case of grand canonical ensemble (GCE) MC simulations. Such a sampling can be carried out by considering the probability of a given configuration of $N$ particles as given by

$$
P\left(N, V, T, \mathbf{s}^{N}\right) \propto P_{0}(N, V, T) \exp (-\beta U) .
$$

The function $P_{0}(N, V, T)$ tunes the number of particles in the sampling. The probability of having a sample configuration with a certain number of particles $N_{i}$ (for given conditions of temperature and volume) can be expressed as

$$
P\left(N_{i}\right)=\frac{P_{0}\left(N_{i}\right) \int d \mathbf{s}^{N_{i}} e^{-\beta U}}{\sum_{N} P_{0}(N) \int d \mathbf{s}^{N} e^{-\beta U}} .
$$

In the particular case of GCE simulations [22,23], one has

$$
P_{0}^{\mathrm{GCE}}(N)=\frac{(z V)^{N}}{N !},
$$

where the fugacity $z$ is related to the chemical potential $\mu$ by $z=\exp (\beta \mu) / \Lambda^{d}$. On the other hand, from Eqs. (37) and (39) we can write 


$$
P\left(N_{i}\right)=\frac{P_{0}\left(N_{i}\right) e^{-\beta F^{\mathrm{ex}}\left(N_{i}\right)}}{\sum_{N} P_{0}(N) e^{-\beta F^{\mathrm{e}^{\mathrm{x}}(N)}} .}
$$

Our aim is to devise a function $P_{0}(N)$ that provides a flat (i.e., uniform) distribution for $P(N)$ over a certain range of densities. In this way we will be able to improve the efficiency of the sampling procedure and attain reliable results for a broad set of thermodynamic conditions. To that purpose we should use $P_{0}(N)=P_{0}^{\text {flat }}(N)$, where

$$
P_{0}^{\text {flat }}(N) \propto \exp \left[\beta F^{\mathrm{ex}}(N)\right], \quad N_{\min } \leqslant N \leqslant N_{\max },
$$

and $P_{0}^{\text {flat }}(N)=0$ otherwise. Of course in most cases $F^{\mathrm{ex}}(N, V, T)$ is not known in advance. In order to estimate this quantity we will exploit the ideas introduced by Wang and Landau [8]. Let us consider a function $\Omega(N)$ related to $P_{0}(N)$ by

$$
P_{0}(N) \propto \exp [\Omega(N)], \quad N_{\min } \leqslant N \leqslant N_{\max } .
$$

In order to estimate $\Omega_{0}(N)=\beta F^{\mathrm{ex}}(N)$, we construct a simulation procedure in which the function $\Omega(N)$ is modified as the simulation proceeds so as to attain a flat histogram of $P(N)$. The numerical operation makes use of a MC simulation that employs standard procedures (periodic boundary conditions, cubic box, etc.) and consists of two main parts that will be referred to as the equilibration run and the sampling run.

The equilibration run is divided into several stages. During these stages $\Omega(N)$ does not remain fixed, but changes so that the system samples the whole predetermined density range. The simplest choice for the initial conditions of the first stage, $s=1$, is $\Omega\left(N_{i}\right)=0$ and $N=0$. On subsequent stages the starting conditions [particle coordinates and $\Omega\left(N_{i}\right)$ values] are chosen to be the final ones of the previous stage.

The computational scheme consists in performing a GCEMC-like simulation [22] in which we use as "external" weighting factor for the particle number sampling Eq. (43) instead of Eq. (40). In this sampling, particle insertions or deletions are selected at random with equal probability. In an insertion, a particle is placed at a random position in the system, while in a deletion the particle to be removed is randomly chosen. The acceptance criterion of a trial configuration is defined by

$$
A(t \mid o)=\max \left[1, \frac{e^{-\beta U_{t}+\Omega\left(N_{t}\right)}}{e^{-\beta U_{o}+\Omega\left(N_{o}\right)}}\right],
$$

where the index $t$ stands for the trial configuration and the index $o$ indicates the current configuration of the system. Once the new configuration is chosen according to Eq. (44), the value of $\Omega\left(N_{n}\right)$ (with $N_{n}$ being the number of particles in the new configuration, which can be either $N_{o}$ or $N_{t}$ ) is updated using

$$
\Omega_{\text {new }}\left(N_{n}\right)=\Omega_{\text {old }}\left(N_{n}\right)-w_{s} .
$$

The correction term $w_{s}$ depends on the stage $s$. After each block of a fixed number of MC moves we check the "flatness" of the overall density distribution at that stage. Let $P_{s}(N)$ be the fraction of configurations with $N$ particles. We consider that the distribution has converged to a "flat" distribution if the following criterion is satisfied:

$$
P_{s}\left(N_{i}\right) \geqslant \frac{1-\delta}{\mathcal{N}_{N}} \quad \forall N_{i}, \quad N_{\min } \leqslant N_{i} \leqslant N_{\max }
$$

where $\mathcal{N}_{N} \equiv N_{\max }-N_{\min }+1$ is the number of densities considered and $\delta(0<\delta<1)$ is a given constant. When Eq. (46) is satisfied, a new simulation stage of the procedure is started, in which the new value of $w$ is given by

$$
w_{s}=\alpha w_{s-1}=\alpha^{s-1} w_{1},
$$

where $0<\alpha<1$. As the procedure advances we expect the running estimates of $P_{0}(N)$ to approach $P_{0}^{\text {flat }}(N)$. Since the changes of $\Omega(N)$ become progressively smaller the system will be drawn near quasiequilibrium simulation conditions and the values of $\Omega(N)-\Omega\left(N_{\text {min }}\right)$ will remain practically constant, thus providing a good approximation to the excess free energy,

$$
\Omega(N) \simeq \beta F^{\mathrm{ex}}(N)+c .
$$

After the completion of an adequate number of stages, we can use the final realization of $\Omega(N)$, denoted $\Omega_{0}(N)$, to determine the weight function $P_{0}\left(N_{i}\right)$-Eq. (43) - and then perform actual equilibrium simulations. The analysis of the results for these equilibrium simulations is carried out by dividing the run into blocks of configurations, which facilitates the estimation of error bars. For each block $k$, we compute the probabilities $P_{k}(N)$ and build the required histograms to evaluate averages of various properties $\left[\langle U(N)\rangle_{k},\left\langle U^{2}(N)\right\rangle_{k}, \ldots\right]$. If $\Omega_{0}(N)$ is close to $\beta F_{\mathrm{ex}}(N)$ [in the sense of Eq. (48)] and an adequate length is chosen for the simulation blocks, we will be able to sample the complete predetermined range of $N$ in each block and then compute the corresponding block estimates of the free energy as

$$
\beta F_{k}^{\mathrm{ex}}(N)=\Omega_{0}(N)-\ln \left[\mathcal{N}_{N} P_{k}(N)\right]+c_{k},
$$

where $c_{k}$ is a constant that can be evaluated if $N_{\min }=0$ by setting $\beta F_{k}^{\mathrm{ex}}(N=0)=0$. Combining the results of several blocks we can extract a global distribution $P(k)$ and evaluate a mean $\beta F^{\mathrm{ex}}$. From $F^{\mathrm{ex}}$ we can estimate the chemical potential and the pressure as a function of the number of particles using

$$
\begin{gathered}
\beta \mu(N)=\frac{1}{2}[\beta F(N+1)-\beta F(N-1)], \\
\beta p(N)=-\beta F(N) / V+N \beta \mu(N) / V .
\end{gathered}
$$

In order to make use of the simulation results obtained at a given temperature to evaluate properties for different temperatures, we have employed a "simplified" reweighting scheme. Along the equilibrium simulations for a given case we store histograms of the quantities $\left\langle U\left(N_{i}\right)\right\rangle$ and $\left\langle U^{2}\left(N_{i}\right)\right\rangle$ as functions of the particle number $N_{i}$. We can then extrapolate the values of $F^{\mathrm{ex}}$ using 


$$
\beta F^{\mathrm{ex}} \simeq\left(\beta F^{\mathrm{ex}}\right)_{0}+U_{0} \Delta \beta-\frac{1}{2}\left(\delta^{2} U\right)_{0}(\Delta \beta)^{2},
$$

where the subscript 0 indicates results at the simulation temperature $T_{0}, \quad U_{0}(N) \equiv\langle U\rangle_{0}, \quad \Delta \beta=\beta-\beta_{0}, \quad$ and $\quad \delta^{2} U \equiv\left\langle U^{2}\right\rangle$ $-\langle U\rangle^{2}$; both $U_{0}$ and $\delta^{2} U$ are functions of $N, V, T$.

In principle, one can estimate the liquid-vapor equilibrium (LVE) at a given temperature by checking if there are two different values of the density with the same values of pressure and chemical potential (Maxwell construction). We found it more straightforward to use a different strategy which yields the same results in the thermodynamic limit. Using the values of the free energy at given conditions of temperature and volume, we can compute the density distribution in the grand ensemble,

$$
P(N \mid \mu) \propto \exp [-\beta F(N)+\beta \mu N],
$$

and determine the value of the chemical potential $\mu_{0}$ that maximizes the density fluctuations. For $\mu_{0}$ we compute the average density $\rho_{m}=\langle N\rangle / V$ and the moments of the density distribution,

$$
m_{i}=\left\langle\left(\rho-\rho_{m}\right)^{i}\right\rangle,
$$

for $i=2,3,4$. We define $\delta \rho=\sqrt{m_{2}}$ and $G=\left(3-m_{4} / m_{2}^{2}\right) / 2$. The moment $m_{3}$ is zero for $\mu=\mu_{0}$ due to the condition of maximum for $m_{2}$. The value of $G$ indicates whether the density distribution is bimodal $(G \approx 1$ in the thermodynamic limit, with equilibrium between two phases with different densities) or normal (one phase, $G \approx 0$ in the thermodynamic limit). In the first case we can estimate the densities of the liquid and vapor phases as $\rho_{\alpha}=\rho_{m} \pm \delta \rho$. The results are expected to show system-size dependence and simulations with different sizes are required to extrapolate the results to the thermodynamic limit.

In order to analyze the critical behavior, FSS methods [24] must be used. A simplified version of the standard treatments was used in this work. The essential difference of our procedure is that we do not take into account the so-called field-mixing phenomenon [24]. The key point that makes our treatment reliable is the fact that the field-mixing contributions to the GCE density distribution at the critical point vanish [24] as the system size $L \equiv V^{1 / 2}$ increases.

Considering that LVE occurs precisely on the $\mu_{0}(T, L)$ line, we expect that as temperature increases along the $\mu_{0}$ line we will find a continuous change from $G \approx 1$ to $G \approx 0$, which will be steeper for larger systems. In the thermodynamic limit, this change takes place abruptly at the critical temperature $T_{c}$. According to FSS theory, at $T_{c}$ the value of $G$ is expected to be system-size independent, $G\left(T_{c}, L\right)=G_{c}$ (for not too small systems), and "universal." For the LVE of systems in two dimensions belonging to the so-called Ising universality class such a value is $G_{c}^{I} \simeq 0.916$ [25]. In order to estimate the critical temperature of the different models we can perform simulations for various system sizes and temperatures, then by means of reweighting techniques we can locate, for each system size, a pseudocritical temperature $T_{c}^{*}(L)$ which satisfies $G\left(T_{c}(L), L\right)=G_{c}$. These pseudocritical temperatures are expected to scale $[24,26,27]$ as

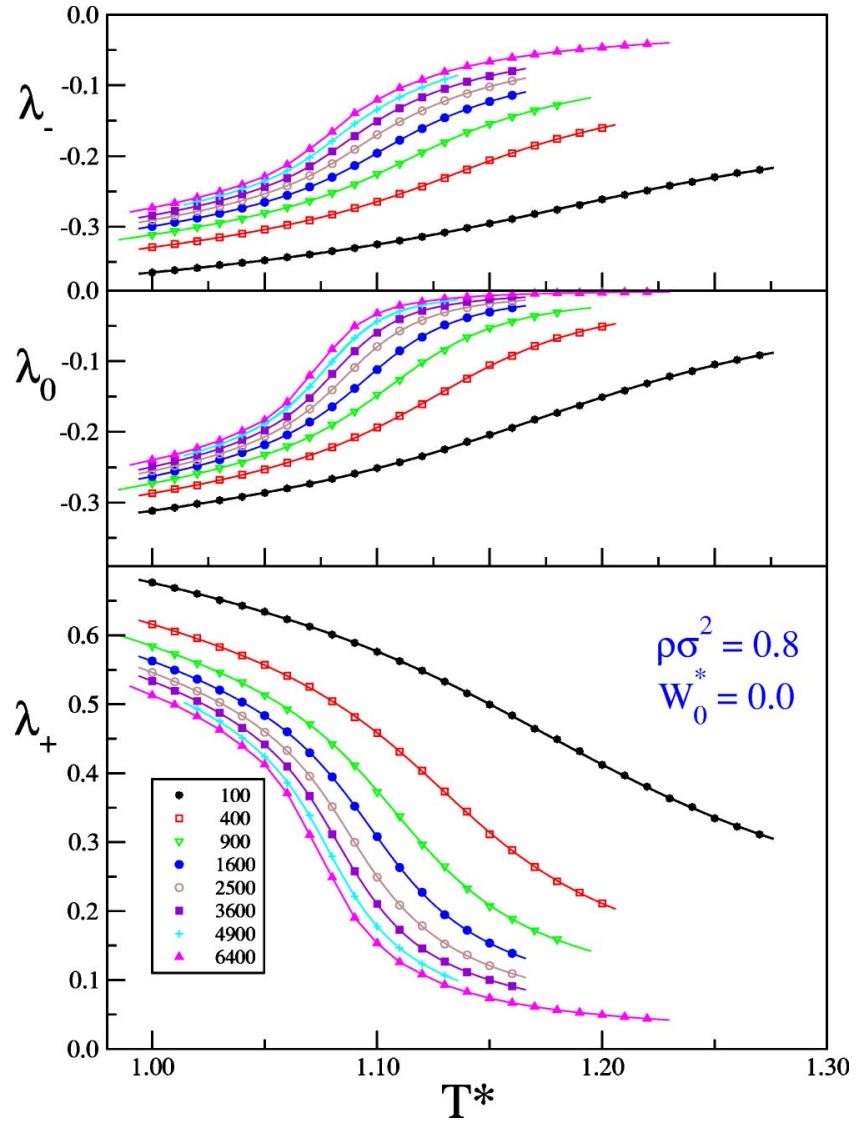

FIG. 1. (Color online) Temperature and size dependence of the eigenvalues of the three-dimensional Saupe tensor for a coplanar Maier-Saupe fluid at $\rho \sigma^{2}=0.8$ without external fields. The orientational order parameter is $S=\lambda_{+}$. Curves are labeled in the legend according to sample size. Symbols denote simulation data and the solid curves are obtained from the histogram reweighting technique.

$$
T_{c}(L)-T_{c} \approx a L^{-b},
$$

with $b=(1+\theta) / \nu, \theta$ and $\nu$ being critical indices. (See $[24,26,27]$ for details.) If $G_{c}$ is unknown for a given system we can still find alternative criteria to define pseudocritical temperatures $T_{c}(L)$, and use them to extrapolate $T_{c}$. If such is the case we expect $b \simeq 1 / \nu[24,26,27]$.

\section{RESULTS}

In our search for the orientational order transition we have considered the following cases: systems with disorienting fields $W_{0}^{*}=1, \infty$ (i.e., planar rotors) and densities $\rho \sigma^{2}$ $=0.8,0.6$, and systems without external field and densities $\rho \sigma^{2}=0.8,0.7,0.6$. In these conditions we have simulated samples from 100 to 6400 particles, generating $10^{4}$ configurations during equilibration and $2.5 \times 10^{5}$ configurations in the production run. Each new configuration for an $\mathrm{N}$-particle sample is generated after $N$ translational move trials and either one cluster move or $N$ single-particle moves.

In Fig. 1 we have plotted the eigenvalues of the threedimensional Saupe's tensor (33) for various sample sizes and temperatures in the absence of external field. Values for the 


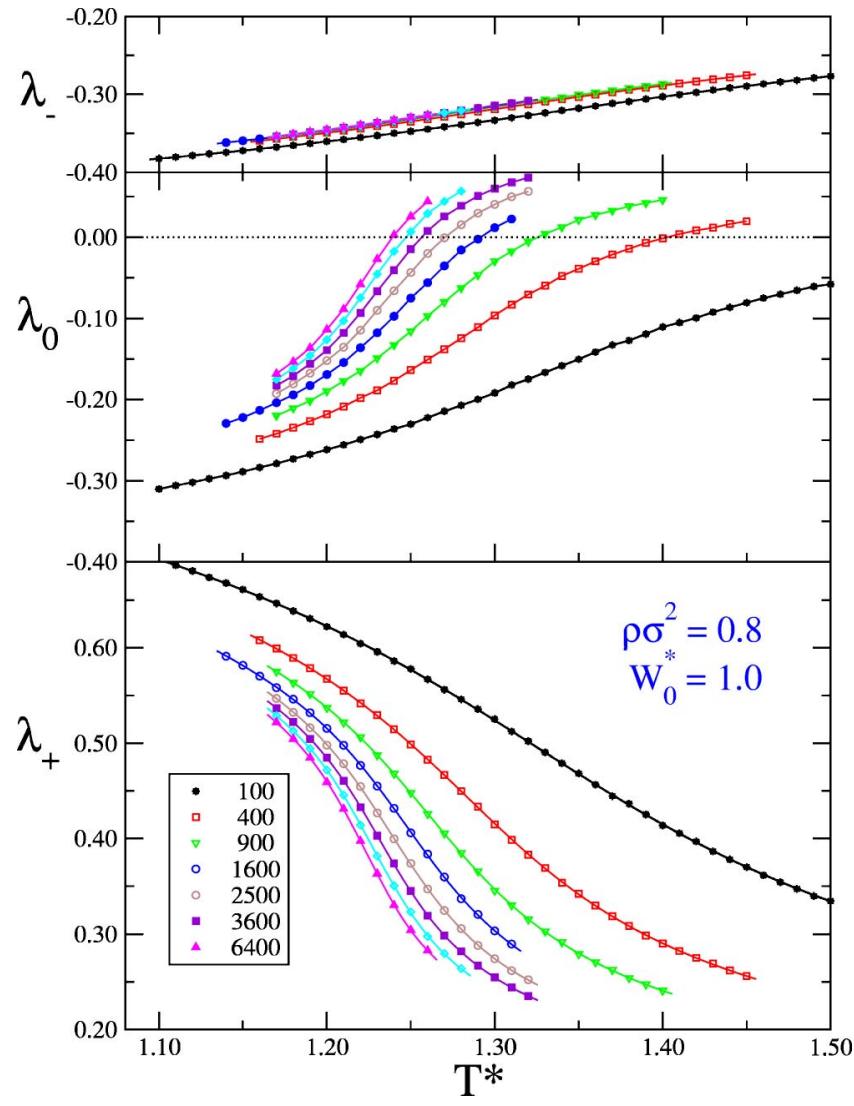

FIG. 2. (Color online) Temperature and size dependence of the eigenvalues of the three-dimensional Saupe tensor for a coplanar Maier-Saupe fluid at $\rho \sigma^{2}=0.8$ in the presence of a perpendicular disorienting field $W_{0}^{*}=1$. Labels as in Fig. 1 .

simulated samples are indicated by symbols; the curves are obtained by means of histogram reweighting. A similar set of results for $W_{0}^{*}=1$ is plotted in Fig. 2. In both cases two interesting features can readily be appreciated. First, one observes that the order parameter $S=\lambda_{+}$(the largest eigenvalue) decreases with the sample size; i.e., one should expect that for an infinite sample this quantity will vanish completely. This is in marked contrast with the behavior of order parameters in ordinary order-disorder transitions, which in the lowtemperature region converge to a finite value. Consequently, in those cases the order parameter curves for various system sizes exhibit a crossover which is not to be found in our results. This vanishing of the low-temperature order parameters in our system is a consequence of a Mermin-Wagnertype theorem $[3,28]$ and signals the likely presence of a BKT transition. However, even if the order parameter vanishes in the thermodynamic limit, it is clear that the slope of the $\lambda_{+}$vs $T^{*}$ curve near a certain transition temperature $T_{\mathrm{KT}}^{*}$ grows when the sample size is increased. This indicates that the fluctuation of the order parameter will exhibit a divergence at the transition. The fact that the order parameter vanishes at temperatures below and above the transition but the slope right at the transition temperature grows with sample size can be easily illustrated by the behavior of an elementary function like $f(x)=\left[1-\tanh \left(N^{2} x\right)\right] / N$ in the vicinity of $x=0$. One clearly observes that this function vanishes in the limit

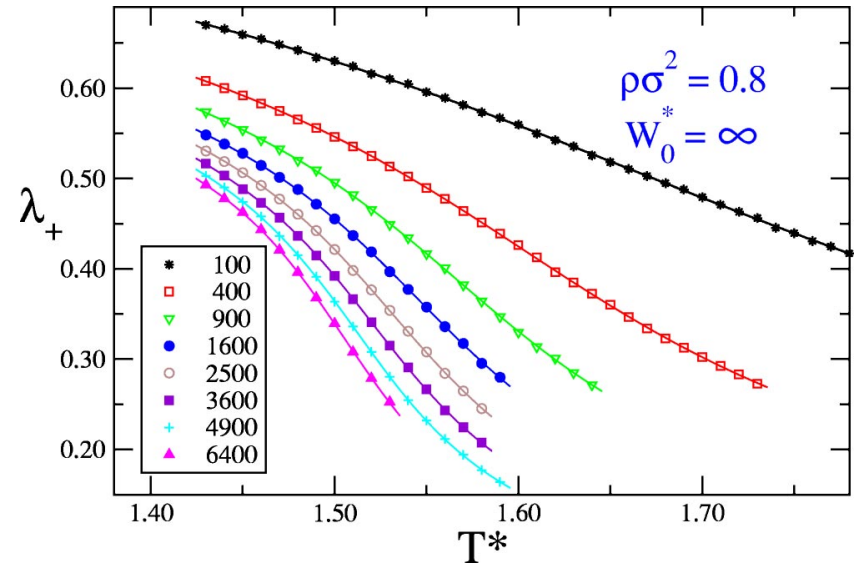

FIG. 3. (Color online) Temperature and size dependence of the eigenvalues of the two-dimensional Saupe tensor for a coplanar Maier-Saupe fluid at $\rho \sigma^{2}=0.8$ in the presence of a perpendicular disorienting field $W_{0}^{*}=\infty$. This is a fully two-dimensional system of planar rotors. Labels as in Fig. 1.

$N \rightarrow \infty$ but its derivative $f^{\prime}(x)=-N \cosh ^{-2}\left(N^{2} x\right)$ exhibits an evident $\delta$-function behavior, essentially the same behavior we will encounter when analyzing the order parameter susceptibility.

Additionally, in Fig. 2 there is another interesting feature. In contrast to the $W_{0}^{*}=0$ case, in the presence of a disorienting field the eigenvalue $\lambda_{0}$ changes sign. Right at the temperature of the sign change one has $\lambda_{-}=-\lambda_{+}$, which is precisely the relation that is satisfied by the eigenvalues of the two-dimensional Saupe's tensor. Moreover, if one calculates the susceptibility from the fluctuation of $\lambda_{+}$,

$$
\chi=N\left(\left\langle\lambda_{+}^{2}\right\rangle-\left\langle\lambda_{+}\right\rangle^{2}\right)
$$

and plots it versus temperature for various sample sizes, one finds that the temperature of the sign change corresponds to the maximum in the susceptibility; i.e., the change of sign in $\lambda_{0}$ signals the order-disorder transition when $W_{0}^{*}>0$. This probably reflects the fact that in the presence of a disorienting field the order-disorder transition is strictly in plane, whereas in the absence of field there is no privileged direction in space as far as the orientation of the particles is concerned. Obviously, in the limiting case $W_{0}^{*}=\infty$ we have a single relevant eigenvalue of the two-dimensional Saupe tensor (33), $\lambda_{+}=-\lambda_{-}$, since we are dealing with a strictly twodimensional system. The corresponding order parameter is plotted in Fig. 3; one can see that both its temperature and sample-size dependence follow the same trends as their finite and zero-field counterparts.

In Fig. 4, together with the susceptibility we plot the constant volume specific heat per particle in units of $k_{B}$, evaluated as

$$
c_{v}=\frac{1}{N T^{* 2}}\left[\left\langle(U / K)^{2}\right\rangle-\langle U / K\rangle^{2}\right] .
$$

In contrast with the susceptibility, this latter quantity does not exhibit any tendency to diverge. This is in accordance with the typical behavior of a BKT transition, in which the 


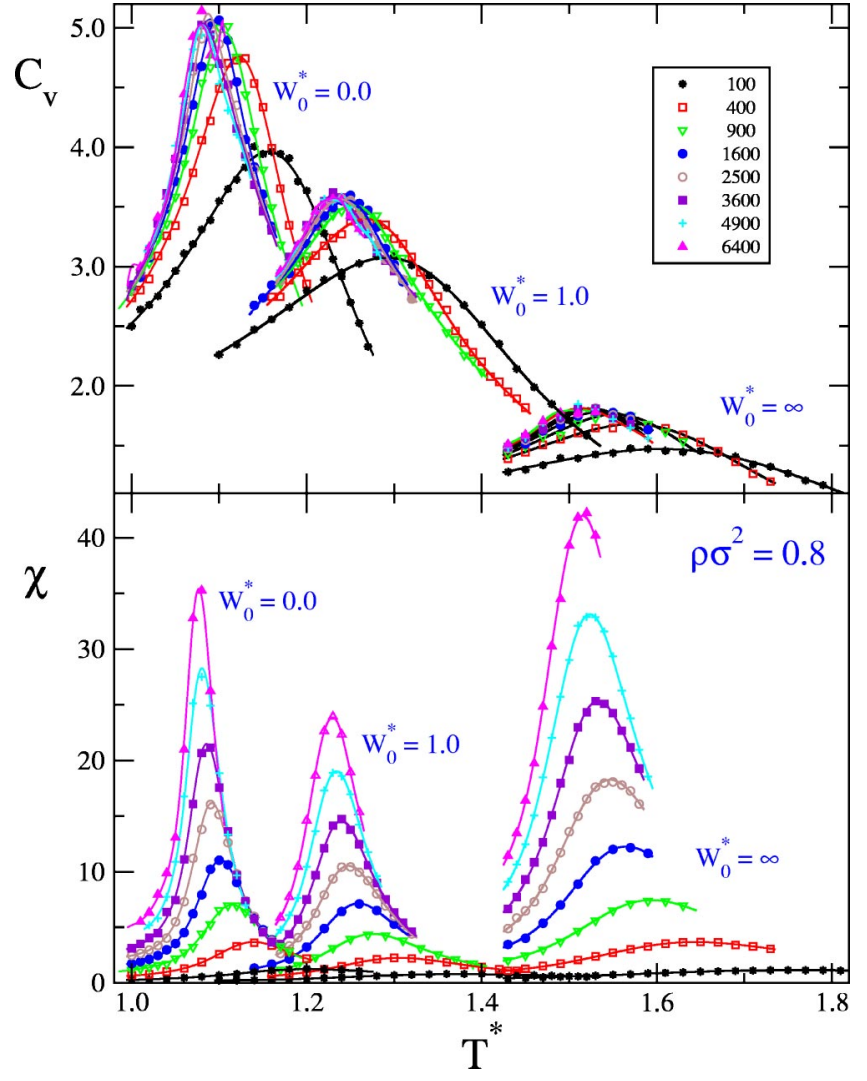

FIG. 4. (Color online) Constant volume specific heat per particle (in units of $k_{B}$ ) and susceptibility (normalized fluctuation of the order parameter $S=\lambda_{+}$) for a coplanar Maier-Saupe fluid at $\rho \sigma^{2}$ $=0.8$. Labels as in Fig. 1 .

specific heat maximum turns into a cusp as the thermodynamic limit is approached [3]. On the other hand, the susceptibility exhibits a clear divergence with increasing sample size and again, as should be expected for a BKT transition, this quantity also diverges for temperatures below the transition temperature. This is evidenced in Fig. 4 by the fact that at low temperatures the value of the susceptibility increases with the sample size. We will see that an estimate of the transition temperature can be obtained from a finite size scaling analysis of the temperatures corresponding to the maxima of the susceptibility, $T_{m}^{*}$.

Additionally, one can resort to a more complete analysis of the order parameter $S$ in terms of Binder's cumulants. Following Weber, Paul, and Binder [29], one can calculate

$$
\begin{gathered}
g_{4}=\left\langle S^{4}\right\rangle /\left\langle S^{2}\right\rangle^{2}, \\
g_{2}=\left\langle S^{2}\right\rangle /\langle S\rangle^{2},
\end{gathered}
$$

the fourth- and second-order cumulants, respectively. These quantities are plotted in Fig. 5. In contrast with the results for the plain order parameter, the cumulant curves do exhibit a clear crossover, in consonance with the behavior found in the usual first- and second-order orientational transitions [13,29]. Now, if we take one sample size (say 100 particles) and analyze the temperatures that correspond to the crossing with curves for other sample sizes, we obtain a series of estimates

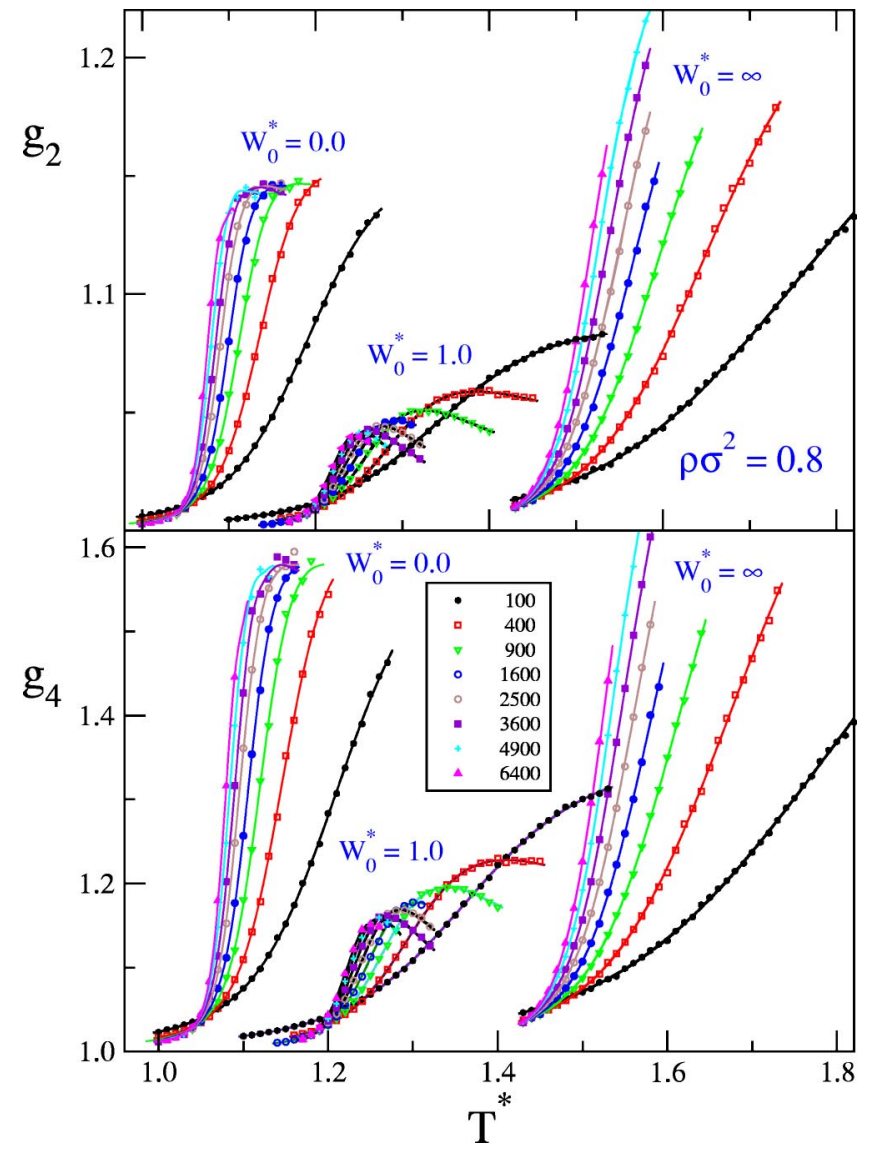

FIG. 5. (Color online) Size and temperature dependence of Binder's cumulants for a coplanar Maier-Saupe fluid at $\rho \sigma^{2}=0.8$. Labels as in Fig. 1.

$T_{\text {cr }}^{*}$ from which is possible to perform a finite size scaling analysis and obtain an estimate of the transition temperature, $T_{\mathrm{KT}}^{*}$. The $T_{m}^{*}$ values obtained from the susceptibility maxima and the crossover temperatures $T_{\text {cr }}^{*}$ must follow the same scaling law. In agreement with previous work [30], we have found that our results follow

$$
T_{\mathrm{KT}}^{*}(l)=T_{\mathrm{KT}}^{*}+\frac{a}{(c+\ln l)^{2}},
$$

where $l=\sqrt{N}$ and $T_{\mathrm{KT}}^{*}(l)$ is either $T_{m}^{*}$ or $T_{\text {cr. }}^{*}$. The fit of $T_{m}^{*}$ values (represented in Fig. 6 vs $1 / \ln N$ ) leads to an estimate of $T_{\mathrm{KT}}^{*}=1.110 \pm 0.008$ when $W_{0}^{*}=1$ and $T_{\mathrm{KT}}^{*}=0.999 \pm 0.001$ when $W_{0}^{*}=0$. A similar fit carried out for the crossings of the $g_{4}$ and $g_{2}$ cumulant curves for 100 and 400 particle samples yields an estimate of $T_{\mathrm{KT}}^{*}=1.13 \pm 0.02$ for $W_{0}^{*}=1$ and $T_{\mathrm{KT}}^{*}$ $=0.99 \pm 0.01$ for $W_{0}^{*}=0$. We can conclude that the transition temperature for $\rho \sigma^{2}=0.8$ and $W_{0}^{*}=0$ is $T_{\mathrm{KT}}^{*}=0.99 \pm 0.01$ and that $T_{\mathrm{KT}}^{*}=1.12 \pm 0.02$ when $W_{0}^{*}=1$. A summary of the results obtained for other densities and disorienting fields is presented in Table I. Note that the results for $\rho \sigma^{2}=0.6$ in the absence of field are somewhat inconclusive since, as shown below, this point lies near the liquid-vapor binodal.

In Fig. 7 we analyze the scaling of the average of the order parameter squared evaluated for the temperatures corresponding to maxima of the susceptibility. According to To- 


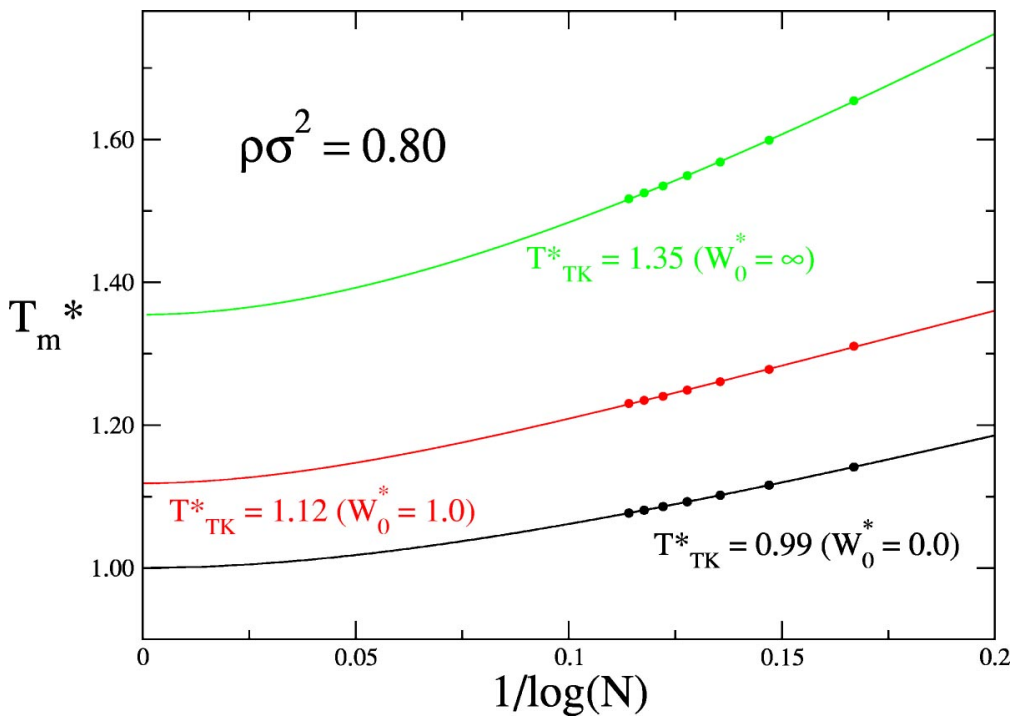

FIG. 6. (Color online) Finite size scaling of the temperature corresponding to a maximum susceptibility, $T_{m}^{*}$, for a coplanar Maier-Saupe fluid at $\rho \sigma^{2}=0.8$ with and without disorienting fields.

mita and Okabe [30], this quantity should scale as

$$
\left\langle S^{2}(l)\right\rangle=\frac{A l^{-\eta}}{(c+\ln l)^{2 r}} .
$$

Since our number of samples is somewhat limited, we simply fit $\left\langle S^{2}(l)\right\rangle \propto l^{-\eta}$ and neglect the logarithmic corrections, which are relatively small in any case [30]. The log-log plot of Fig. 7, and similar calculations for other densities and fields, lead to the values of $\eta$ collected in Table I. One observes that the zero-field and the infinite-field values yield similar results for all densities, whereas the values for $W_{0}^{*}$ $=1$ are significantly lower. Interestingly, Kunz and Zumbach [3] give $\eta=0.4$ for the lattice version of our model without disorienting field, and Tomita and Okabe give $\eta=0.24$ for the $X Y$ model, which is essentially our model when $W_{0}^{*}$ $\rightarrow \infty$. Our calculations, however, predict a minimum $\eta$ for finite nonzero field and do not show a substantial decrease in the critical exponent in the fully coplanar sample. We do not have a clear explanation for these discrepancies, and they might be ascribed to the fact that our largest sample sizes are relatively modest compared with those of lattice calculations (our largest $l=80$ vs $l=256$ in Ref. [3] and 512 in [30]). Only the zero-field continuum and lattice results seem to agree qualitatively.

The lack of true long-range order is also reflected in the behavior of the angular correlation function $G_{2}(r)$ which

TABLE I. Transition temperatures and critical exponent $\eta$ for the planar Maier-Saupe fluid with and without a disorienting field.

\begin{tabular}{|c|c|c|c|c|c|c|}
\hline \multirow[b]{3}{*}{$\rho \sigma^{2}$} & \multicolumn{6}{|c|}{$W_{0}^{*}$} \\
\hline & \multicolumn{2}{|c|}{0} & \multicolumn{2}{|c|}{1} & \multicolumn{2}{|c|}{$\infty$} \\
\hline & $T_{K T}^{*}$ & $\eta$ & $T_{K T}^{*}$ & $\eta$ & $T_{K T}^{*}$ & $\eta$ \\
\hline 0.6 & $0.51(3)$ & $0.29(3)$ & $0.65(2)$ & $0.12(3)$ & $0.79(2)$ & $0.27(1)$ \\
\hline 0.7 & $0.78(1)$ & $0.36(2)$ & & & & \\
\hline 0.8 & $0.99(1)$ & $0.38(2)$ & $1.12(2)$ & $0.17(2)$ & $1.35(1)$ & $0.32(1)$ \\
\hline
\end{tabular}

measures the relative orientation of two spins separated a distance $r$ as is defined by

$$
G_{2}(r)=5\left\langle P_{2}\left(\cos \theta_{12}\right)\right\rangle_{r}
$$

where $\langle\cdots\rangle_{r}$ denotes the ensemble average in a spherical shell contained between $r-\Delta r / 2$ and $r-\Delta r / 2$ with a sufficiently small $\Delta r$. This quantity is plotted in Fig. 8 in the absence of external field for a low-temperature state well below the transition temperature and various sample sizes. One clearly observes that the sample-size dependence becomes apparent for the smallest sample around $5 \sigma$ but for a regular size of 900 particles the results up to $10 \sigma$ are reliable. In accordance with the behavior of the order parameter, one would get $\lim _{r \rightarrow 0} G_{2}(r)=0$ in the thermodynamic limit. However, what is evident from Fig. 8 is that the system exhibits a large degree of orientational order in the short and medium range. This order is destroyed at larger distances by the presence of collective excitations, spin waves that on the other hand allow for coherent orientation of the spins at smaller length scales [31]. Another possibility is the formation of vortices that would destroy the long-range order, as recently found in ferrofluid monolayers [32]. In contrast, when both the disorienting field $\mathbf{W}_{0}$ and a transverse field $\mathbf{A}_{0}$, like the one used in [1], are turned on, these extreme size dependencies disappear, since then the system exhibits a true long-range nematic order induced by the fields.

In our study of the gas-liquid transition we have simulated several models with different values of $W_{0}^{*}$ [Eqs. (1)-(3)]: $W_{0}^{*}=0, W_{0}^{*} \rightarrow-\infty$ (simple isotropic Yukawa-like interaction), $W_{0}^{*}=-1, W_{0}^{*}=+1$, and $W_{0}^{*} \rightarrow \infty$ (orientations constrained in a plane). In the limit $W_{0}^{*} \rightarrow-\infty$, the spins are oriented in the same direction and the plain radial $u_{0}(r)$ interaction is recovered. As mentioned earlier, in the limit $W_{0}^{*} \rightarrow \infty$, the system resembles a continuum version of the lattice gas $X Y$ model, with an added attractive interaction.

The starting point for the simulations in the equilibration part is a system without particles. The simulation runs are arranged in cycles. In each cycle we first perform a certain number of particle insertion or deletion attempts, then we 


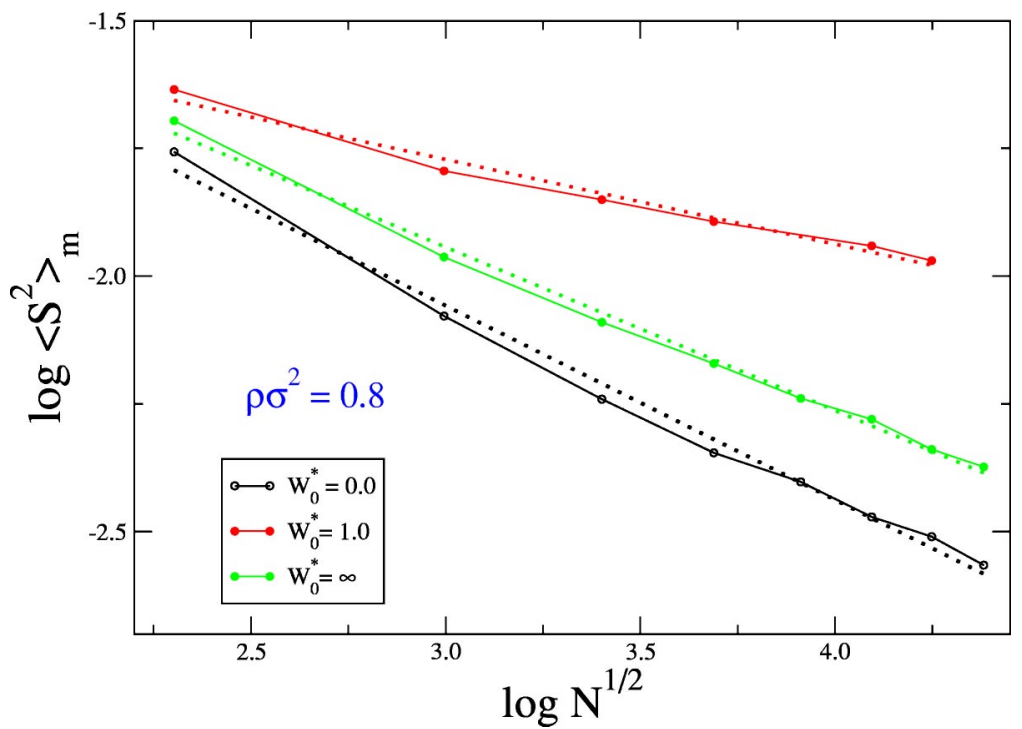

FIG. 7. (Color online) Finite size scaling of the average of the order parameter squared at a temperature corresponding to a maximum susceptibility $\left(\left\langle S^{2}\right\rangle_{m}\right)$ for a coplanar Maier-Saupe fluid at $\rho \sigma^{2}=0.8$ with and without disorienting fields. Solid lines are drawn as a guide to the eye and dotted lines represent the corresponding least-squares fits.

perform $N$ one-particle translation attempts and finally $N$ one-particle orientational moves. In the first instance we choose with equal probability to perform either a particle insertion or a particle deletion. The insertion attempt is rejected if the current number of particles $N$ is equal to $N_{\text {max }}$; in other cases, we check whether the position of the new particle (chosen at random with uniformly distributed probability over the volume of the box) is forbidden due to hardsphere overlaps and finally (if required) we generate at random the orientation of the new particle, compute the change of the potential energy, and apply the acceptance criterion given in Eq. (44). The deletion attempts are rejected if $N \leqslant N_{\text {min }}$. In other cases a particle is chosen at random and the deletion is accepted with the probability given by (44). We typically use 5000-20000 insertion or deletion moves per cycle (with more attempts for larger systems). The translational moves are performed using the standard MC techniques [22]. The orientational changes are performed by choosing the trial orientation at random. In these two latest types of move, the Metropolis criterion [22] is used to accept or reject the trial configurations.
After some preliminary tests, we typically run the simulations for several temperatures around and below the expected LVE critical temperatures using various system sizes. The sample volumes were set to $L^{2} \sigma^{2}$, with $L$ lying in the range between 10 and 40 . In all the cases we have used in the Wang-Landau runs the values $w_{1}=0.01, \alpha=1 / \sqrt{2}$, and $\delta$ $=0.1$. We typically used 20-25 stages to obtain an initial estimation of $\Omega_{0}(N)$.

The LVE critical points were estimated using the simplified reweighting procedure and FSS analysis explained in the previous section. The pseudocritical temperatures were chosen to be those that make $G$ equal to the critical value for the two-dimensional (2D) Ising universality class. In order to estimate the critical temperatures we have performed leastsquares fittings of $T_{c}^{*}(L)$ as a function of $L^{-1}$,

$$
T_{c}^{*}(L)=T_{c}^{*}+a_{t}\left(\frac{1}{L}\right)^{b_{t}},
$$

where $T_{c}^{*}, a_{t}$, and $b_{t}$ are free parameters. The use in Eq. (62) of three parameters takes into account the possible deviations

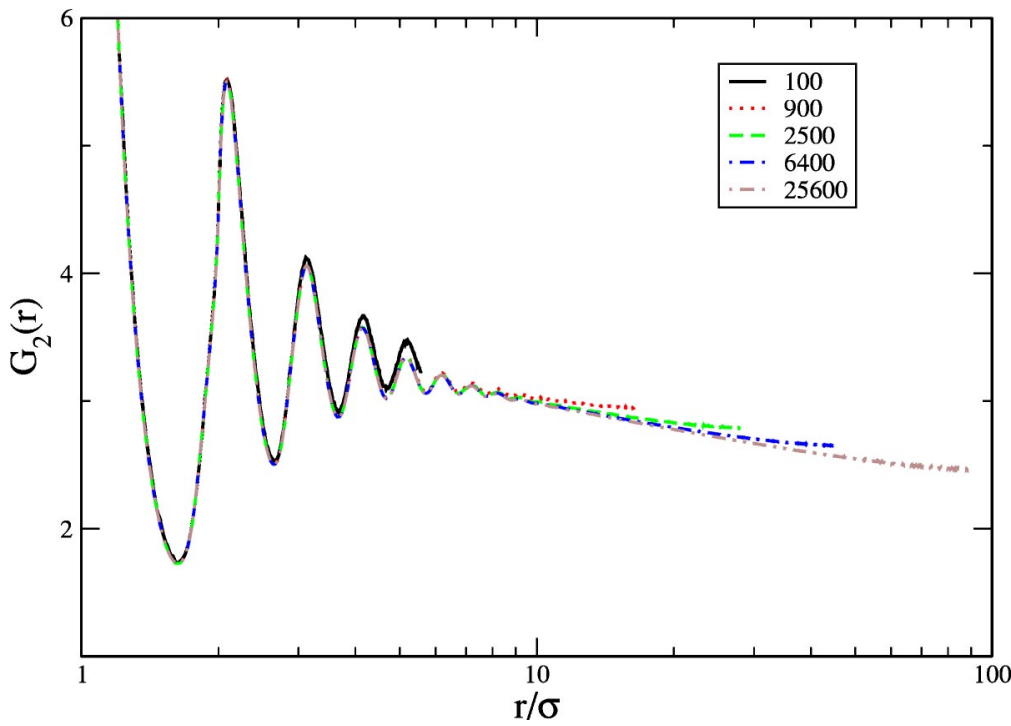

FIG. 8. (Color online) Size dependence of the angular correlation $G_{2}(r)$ in the absence of external field for the planar nematogenic liquid at $\rho \sigma^{2}=0.8$ and $T^{*}=0.7$. Notice the $\log _{10}$ scale of the abscissa. 
TABLE II. Pseudocritical temperatures $T_{c}^{*}(L)$ for different system sizes and different models (see the text for details).

\begin{tabular}{rrrrrr}
\hline \hline & \multicolumn{5}{c}{$W_{0}^{*}$} \\
$L / \sigma$ & 0 & 1 & $\infty$ & -1 & $-\infty$ \\
\hline 10 & 0.486 & & & & \\
12 & 0.496 & 0.501 & 0.550 & 0.510 & 0.645 \\
15 & 0.505 & & & & \\
16 & 0.507 & 0.509 & 0.558 & 0.514 & 0.650 \\
20 & 0.515 & 0.514 & 0.563 & 0.516 & 0.653 \\
24 & 0.520 & 0.517 & 0.566 & 0.518 & 0.654 \\
25 & 0.520 & & & & \\
28 & 0.522 & 0.518 & 0.568 & 0.518 & 0.655 \\
32 & 0.525 & 0.520 & 0.569 & 0.519 & 0.655 \\
36 & 0.526 & 0.521 & 0.570 & 0.519 & 0.656 \\
40 & 0.528 & 0.521 & 0.571 & 0.519 & 0.656 \\
\hline \hline
\end{tabular}

from the scaling laws of the smallest systems and the fact that the systems with orientation-dependent interactions could exhibit a critical behavior different from that of the simple 2D Yukawa fluid (which is expected to belong to the 2D Ising universality class), since the influence of a continuous BKT transition on the gas-liquid critical behavior is not known a priori. In Table II we gather the results of the pseudocritical temperatures. Once $T_{c}^{*}$ is estimated using Eq. (62), we use the results of different system sizes at $T_{c}^{*}$ to extrapolate $\rho_{c}$ and $\beta p_{c}$. In Table III we show the results for the models considered in this work.

In order to estimate the LVE we have first used the simulation results and reweighting techniques introduced in the previous section to determine the values $\rho_{m}$ and $\delta \rho$ for different subcritical temperatures and system sizes. In some of the systems there is a significant size dependence of the coexisting vapor and liquid densities, therefore we have extrapolated the values to the thermodynamic limit, fitting the results for various system sizes (at a given temperature) to the expressions

$$
\begin{aligned}
& \rho_{m}(T, V)=\rho_{m}(T)+\sum_{k=1}^{k_{\max }} a_{k}(1 / V)^{k}, \\
& \delta \rho(T, V)=\delta \rho(T)+\sum_{k=1}^{k_{\max }} b_{k}(1 / V)^{k},
\end{aligned}
$$

where $k_{\max }$ is chosen in each particular case to be either 1 or 2 , depending on the behavior of the corresponding property with the system size. It is clear, however, that this extrapolation scheme, especially that of Eq. (64), can become inaccurate for temperatures close to the critical point. In order to draw the envelope of the liquid-vapor coexistence region we have fitted the values of $\rho_{m}(T)$ and $\delta \rho\left(T^{*}\right)$ to

$$
\rho_{m}(T) \sigma^{2}=\sum_{i=0} c_{i}\left(T^{*}\right)^{i}
$$

$$
\ln \left[\delta \rho(T) \sigma^{2}\right]=\ln A+b \ln \left(T_{c}^{*}-T^{*}\right) .
$$

In Eq. (65) we have used two or three coefficients. In Eq. (66) $T_{c}^{*}$ is not fitted, but taken from the previous critical point estimates. On the other hand, in the fitting of Eq. (66) we only retain data from states not too close to the critical temperature (in practice $T^{*} \leqslant 0.95 T_{c}^{*}$ ). Therefore, one should not deem these results for the parameter $b$ as reliable estimates of the corresponding critical exponent. We have also checked the behavior of the values of $\delta \rho\left(T_{c}^{*}\right)$ as a function of the system size. The prediction from FSS theory is

$$
\delta \rho\left(T_{c}^{*}, L\right) \sim L^{-\beta / \nu},
$$

with $\beta / \nu=0.125$ for the 2D Ising universality class. We have fitted the results of $\delta \rho\left(T_{c}^{*}, L\right)$ to the expression

TABLE III. Estimates of critical properties and liquid-vapor equilibrium (see the text for details); $T_{\text {low }}^{*}$ represents the lowest temperatures used in the fitting of the liquid-vapor binodal.

\begin{tabular}{cccccc}
\hline \hline$W_{0}^{*}$ & 0 & +1 & $+\infty$ & $-\infty$ & -1 \\
\hline$T_{c}^{*}$ & $0.542(3)$ & $0.5256(20)$ & $0.5765(20)$ & $0.6572(3)$ & $0.5199(10)$ \\
$\rho_{c} \sigma^{2}$ & $0.524(10)$ & $0.475_{-0.010}^{+0.060}$ & $0.47_{-0.02}^{+0.08}$ & $0.360(1)$ & $0.412(1)$ \\
$p_{c} \sigma^{2} / k_{B} T$ & $0.54(1)$ & $0.300(6)$ & $0.220(2)$ & $0.067(1)$ & $0.14(1)$ \\
$b_{t}$ & $1.04(10)$ & $1.45(17)$ & $1.3(2)$ & $2.0(2)$ & $2.0(4)$ \\
$c_{0}$ & 0.300 & 0.326 & 0.890 & 0.674 & 0.450 \\
$c_{1}$ & 0.402 & 0.270 & -1.941 & -0.710 & -0.0745 \\
$c_{2}$ & & & 2.048 & 0.353 & \\
$A$ & 0.918 & 0.771 & 0.727 & 0.554 & 0.629 \\
$b$ & 0.492 & 0.341 & 0.322 & 0.192 & 0.213 \\
$T_{\text {low }}^{*}$ & 0.450 & 0.440 & 0.490 & 0.530 & 0.450 \\
$b_{\delta} \sim \beta / \nu$ & $0.28(4)$ & $0.19(3)$ & $0.21(3)$ & $0.14(1)$ & $0.14(3)$ \\
\hline \hline
\end{tabular}




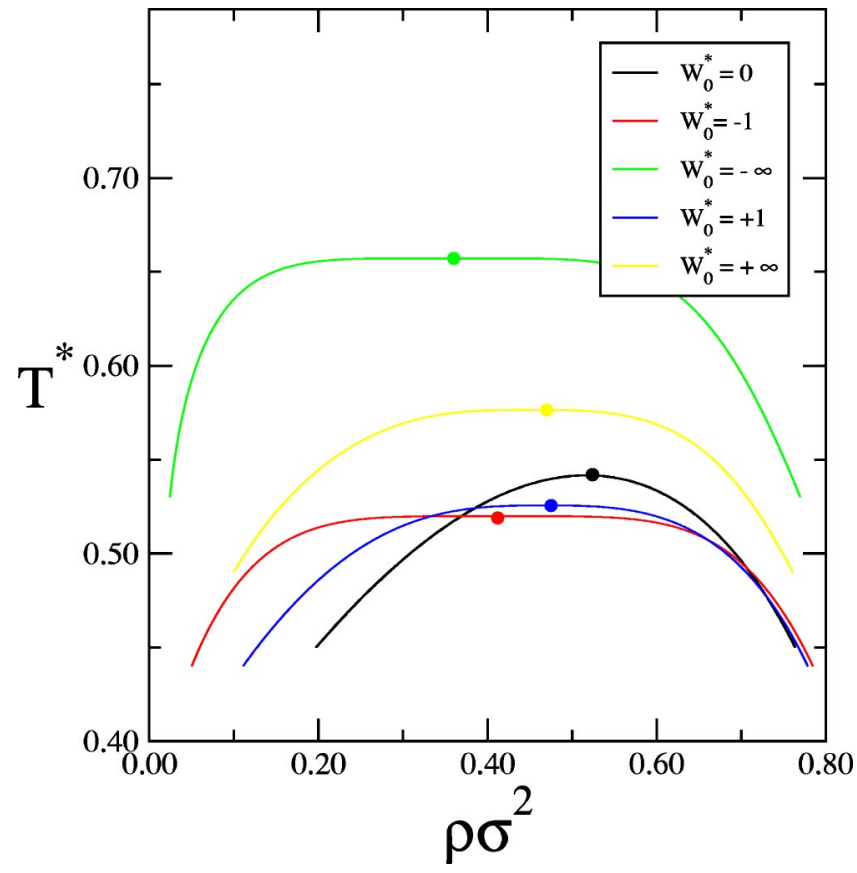

FIG. 9. (Color online) Liquid-vapor equilibria of the different models. The symbols show the estimates of the critical points; the critical temperatures of the different models satisfy $T_{c}^{*}\left(W_{0}^{*}=-1\right)$ $<T_{c}^{*}\left(W_{0}^{*}=1\right)<T_{c}^{*}\left(W_{0}^{*}=0\right)<T_{c}^{*}\left(W_{0}^{*}=\infty\right)<T_{c}^{*}\left(W_{0}^{*}=-\infty\right)$.

$$
\ln \left[\delta \rho\left(T_{c}^{*}, L\right) \sigma^{2}\right]=a_{\delta}-b_{\delta} \ln L
$$

In Table III we collect the results for the different fits. The corresponding phase diagrams are plotted in Fig. 9 and in Fig. 10 the $W_{0}^{*}=0,1$, and $\infty$ LVE curves are plotted together with the estimates of the BKT transition. The models with $W_{0}^{*}=-\infty$ (Yukawa) and $W_{0}^{*}=-1$ seem to show a typical Isinglike critical behavior-with all the cautions regarding the critical parameter fitting commented upon above. In contrast, the models with $W_{0}^{*} \geqslant 0$ depart from the Ising-like behavior probably due to the presence of tricritical points or critical end points where the LVE line meets the orientational transition line. Obviously, the limitations of present-day simulation procedures do not allow for an assessment of the nature of the meeting point of the two types of transition. The results are compatible with the presence of either a tricritical point or a critical end point. On the other hand, the results of the FSS analysis do show significant differences between the critical behavior of the Maier-Saupe model with $W_{0}^{*}=0$ and those systems with $W_{0}^{*}>0$. In the latter case the critical behavior resembles to some extent that of the Blume-Capel model [33]. Finally, the relatively large error bars in the estimations of the critical densities of models with $W_{0}^{*} \geqslant 0$ suggest the possible nonanalyticity of $\rho_{m}\left(T_{c}^{*}\right)$ in the vicinity of the critical point; preliminary results [34] using related lattice-gas models seem to support this hypotheses. Nevertheless, the precise nature of the critical behavior of these models is beyond the scope of this work, and a proper study of these issues would certainly require the consideration of larger and simpler systems, like the corresponding lattice gas models. We are currently working in this direction. In addi-

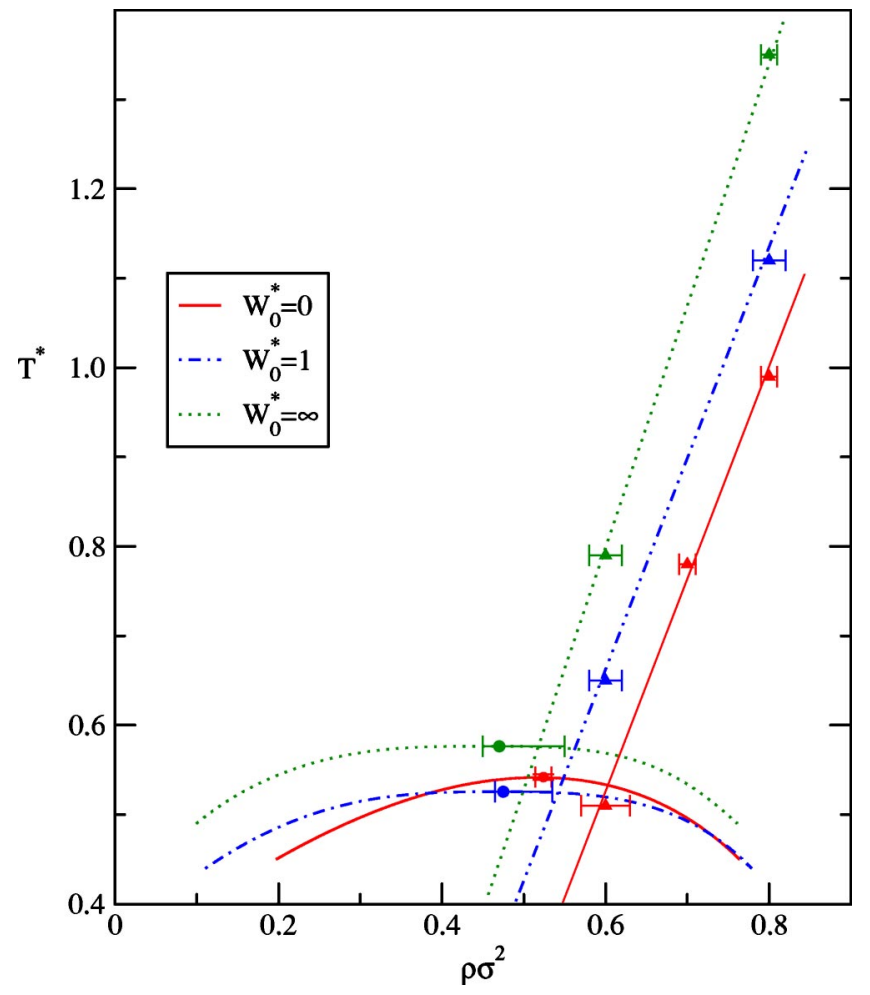

FIG. 10. (Color online) Simulation results for the phase diagram of the planar nematogenic liquid for various disorienting fields. Filled circles indicate the location of the gas-liquid critical point estimates. Filled triangles denote the location of the BKT orderdisorder transition for various densities. These points are connected with straight lines which separate the fully isotropic phase from the BKT ordered phase.

tion to the change in the gas-liquid critical behavior induced by the presence of the external field, we also observe an interesting feature in the field dependence of the critical temperatures. First, one notices that when the field is turned on the critical temperature is lowered (and this is so for both positive and negative fields). Then, when the field is further increased towards $\infty$ or $-\infty$ the critical temperature rises. This latter feature is easily understood since in both cases the increase in the field implies a larger net attraction-when $W_{0}^{*}=-\infty$ since the spins are all parallel and perpendicular to the plane and when $W_{0}^{*}=\infty$ since the angular interaction (3) in two dimensions has a nonzero attractive angular average. This increase in the net attraction also explains why the width of the binodal increases uniformly as the field is augmented. As to the reduction in the critical temperature for small fields, it could well be due to the change in the universality class when the field is turned on. A similar feature was found by Sokolovska and Sokolovskii [35] in a threedimensional Heisenberg model in their anisotropic mean spherical approximation (MSA) calculations. In particular, the situation described in Ref. [35] is similar to our negative disorienting field calculations.

We focus now on what the theory can tell us regarding these systems. As mentioned before, the anisotropic MSA of [36] leads in the zero-field case to a first-order transition in which the gas-liquid transition is coupled to a nematic- 
isotropic transition and, as the temperature is raised, the density jump of the transition shrinks, eventually disappearing at very high temperatures. In the case of an infinite disorienting field we are dealing with the $X Y$ model, which is also known to exhibit a BKT transition [30,31]. Now, the anisotropic MSA predicts in this limit a second-order transition $[4,5]$. Since both the MSA and the density functional theory (DFT) are essentially mean field approaches [the nonlinear correction of the modified mean field (MMF) does not radically change the general behavior of the approximation] we will encounter again the same situation as in Refs. [4,5,36], as illustrated in Fig. 11. If we compare now Figs. 10 and 11 it is clear that the disorienting field shifts the order-disorder transition to higher temperatures and that the gas-liquid equilibrium curve is displaced to lower densities; i.e., the location of what would be the gas-liquid critical point in an ordinary condensation moves toward substantially lower densities and slightly higher temperature. The simulation results indicate a slight decrease in the gas-liquid critical temperature for $W_{0}^{*}$ $=1$ (cf. Table II) which cannot be appreciated in the DFT results, in which both the order-disorder and the gas-liquid transition are smoothly coupled into a first-order transition. Finally, the DFT in the infinite field limit predicts the presence of a tricritical point in both the MMF and the MF approaches. Our simulation results for the order-disorder transition in this latter instance, in accordance with findings for the $X Y$ model $[30,31]$ also indicate the presence of a BKT transition line that meets the LVE curve either at a tricritical point or at a critical end point. As a final consideration regarding the two DFT approximations here employed, one observes that both lead to similar results, with the MMF being slightly superior, in part due to a better treatment of the isotropic phase.

In summary, we have presented a detailed simulation study of the phase behavior of a Maier-Saupe fluid whose particles are constrained to lie in a plane, with and without an external disorienting field. The simulation results confirm the presence of an order-disorder BKT transition that at low temperatures couples with a gas-liquid transition. The DFT approach reproduces qualitatively the field dependence of the phase behavior but predicts a first-order order-disorder transition that gradually becomes second order as the magnitude of the disorienting field is increased. The DFT as constructed here is unable to properly describe a BKT order-disorder transition.

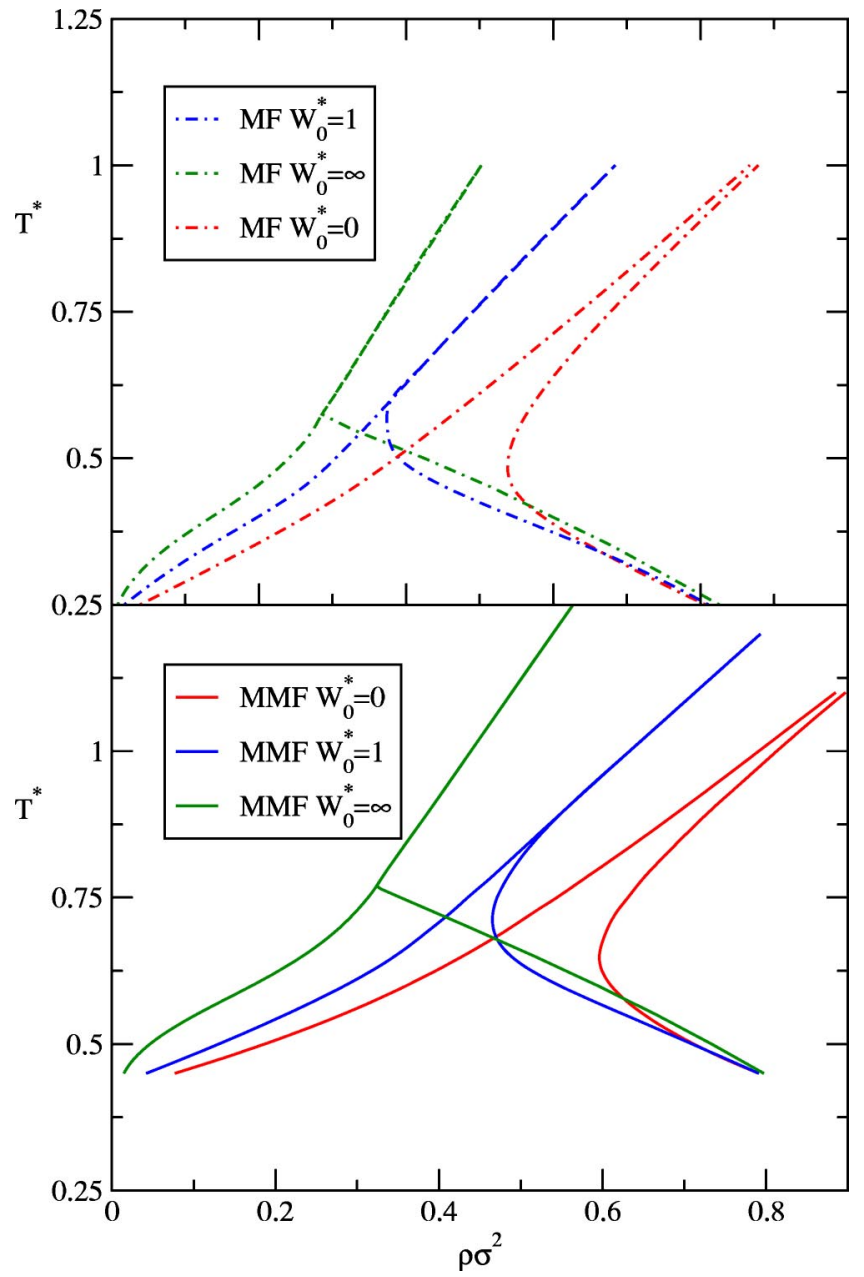

FIG. 11. (Color online) Phase diagram of the planar nematogenic liquid for various disorienting fields in the MF and MMF approximations.

\section{ACKNOWLEDGMENT}

The financial support of the Dirección General de Investigación Científica is acknowledged by E.L, C.M., and N.G.A. under Grant No. FIS2004-02954-C03-01.
[1] F. Lado, E. Lomba, and C. Martín, J. Mol. Liq. 112, 51 (2004); F. Lado, E. Lomba, C. Martín, and N. G. Almarza, J. Phys. Condensed Matter (to be published).

[2] W. Maier and A. Saupe, Z. Naturforsch. A 14, 882 (1959).

[3] H. Kunz and G. Zumbach, Phys. Rev. B 46, 662 (1992).

[4] T. G. Sokolovska, R. O. Sokolovskii, and M. F. Holovko, Phys. Rev. E 62, 6771 (2000).

[5] T. G. Sokolovska, R. O. Sokolovskii, and M. F. Holovko, Phys. Rev. E 64, 051710 (2001).

[6] V. L. Berezinskii, Sov. Phys. JETP 32, 493 (1971).

[7] J. M. Kosterlitz and D. J. Thouless, J. Phys. C 6, 1181 (1973).
[8] F. Wang and D. P. Landau, Phys. Rev. Lett. 86, 2050 (2001); Phys. Rev. E 64, 056101 (2001).

[9] A. M. Ferrenberg and R. H. Swendsen, Phys. Rev. Lett. 63, 1195 (1989); R. H. Swendsen, Physica A 194, 53 (1993).

[10] C. Holm and W. Janke, Phys. Rev. B 48, 936 (1993).

[11] R. H. Swendsen and J. S. Wang, Phys. Rev. Lett. 58, 86 (1987).

[12] N. V. Priezjev and R. A. Pelcovits, Phys. Rev. E 63, 062702 (2001).

[13] E. Lomba, C. Martín, and N. G. Almarza, Mol. Phys. 101, 1667 (2003). 
[14] R. Evans, Adv. Phys. 28, 143 (1979).

[15] S. Luding, Phys. Rev. E 63, 042201 (2001).

[16] E. Lomba, J. J. Weis, and C. F. Tejero, Phys. Rev. E 58, 3426 (1998).

[17] W. H. Press, B. P. Flannery, S. A. Teukolsky, and W. T. Vetterling, Numerical Recipes (Cambridge University Press, Cambridge, U.K., 1990).

[18] P. I. Teixeira and M. M. Telo da Gama, J. Phys.: Condens. Matter 3, 111 (1991).

[19] M. S. Shell, P. G. Debenedetti, and A. Z. Panagiotopoulos, Phys. Rev. E 66, 056703 (2002).

[20] Q. Yan, R. Faller, and J. J. de Pablo, J. Chem. Phys. 116, 8745 (2002).

[21] Q. Yan and J. J. de Pablo, Phys. Rev. Lett. 90, 035701 (2003).

[22] M. P. Allen and D. J. Tildesley, Computer Simulation of Liquids (Clarendon, Oxford, 1987).

[23] D. Frenkel and B. Smit, Understanding Molecular Simulation, 2nd ed. (Academic, London, 2002).

[24] N. B. Wilding, Phys. Rev. E 52, 602 (1995).
[25] Y. Tomita and Y. Okabe, Phys. Rev. B 66, 180401(R) (2002).

[26] A. D. Bruce, J. Phys. A 18, L873 (1985).

[27] J. J. Weis, D. Levesque, and J. M. Caillol, J. Chem. Phys. 109, 7486 (1998).

[28] N. D. Mermin and H. Wagner, Phys. Rev. Lett. 17, 1133 (1966).

[29] H. Weber, W. Paul, and K. Binder, Phys. Rev. E 59, 2168 (1999).

[30] Y. Tomita and Y. Okabe, Phys. Rev. B 65, 184405 (2002).

[31] A. I. Fariñas-Sanchez, R. Paredes, and B. Berche, Phys. Lett. A 308, 461 (2003).

[32] E. Lomba, F. Lado, and J. J. Weis, Phys. Rev. E 61, 3838 (2000).

[33] N. B. Wilding and P. Nielaba, Phys. Rev. E 53, 926 (1996).

[34] N. G. Almarza (unpublished).

[35] T. G. Sokolovska and R. O. Sokolovskii, Phys. Rev. E 59, R3819 (1999).

[36] T. G. Sokolovska, M. F. Holovko, and R. O. Sokolovskii, Ukr. Fiz. Zh. 42, 1304 (1997). 